\title{
Pregnancy and parental leave among plastic surgery residents in Canada: a nationwide survey of attitudes and experiences
}

\author{
Haley Augustine, MD, MScOT \\ Syed Ali Rizvi, MD \\ Emily Dunn, MKin \\ Jessica Murphy, MSc \\ Helene Retrouvey, MD, MSc \\ Johnny lonut Efanov, MD \\ Anna Steve, MD \\ Becher Alhalabi, MD, MHPE \\ Ronen Avram, MD, MSc \\ Sophocles Voineskos, MD, MSc
}

Accepted Dec. 5, 2019

\section{Correspondence to:}

H. Augustine

McMaster Children's Hospital

McMaster University

1280 Main St W

Hamilton ON L8N $3 Z 5$

haley.augustine@gmail.com

DOI: $10.1503 /$ cjs.004919

\begin{abstract}
SUMmARY
Small surgical residency programs like plastic surgery can be challenging environments to accommodate parental leave. This study aimed to report the experiences, attitudes and perceived support of Canadian plastic surgery residents, recent graduates and staff surgeons with respect to pregnancy and parenting during training. Residents and staff surgeons were invited via email to participate in an online survey. The results presented here explore experiences of pregnancy and parental leave of current plastic surgery residents and staff surgeons. Residents' and staff surgeons' perceptions of program director support, policies, negative comments and the impact of parental leave on the workload of others were also explored. Although the findings suggest that there may be improvements in the support of program directors, there continues to be a negative attitude in surgical culture toward pregnancy during residency. The perceived confusion of respondents with respect to programspecific policies emphasizes the need for open conversations and standardization of parental leave.
\end{abstract}

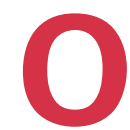

$\mathrm{n}$ average, following the completion of a surgical residency, exiting trainees in Canada are 33.2 years of age. ${ }^{1}$ The average age at which mothers in Canada had their first child was 29.2 years in $2016 .{ }^{2}$ Therefore, residents who hope to start a family may consider pregnancy during their training. However, surgical residents are in an undesirable position to start a family because of their long work hours, the physically demanding nature of their work, specific occupational hazards and less flexibility in residency rotation scheduling. ${ }^{3}$ A survey of members and candidates of the American Society of Plastic Surgeons found that $72.6 \%$ of women and $39.2 \%$ of men delayed having children because of the demands of training. ${ }^{4}$ As there is an increased risk of both maternal and fetal complications related to deferred childbirth, especially after age 35, many plastic surgery graduates may find themselves conflicted between the demands of a professional career and parenting priorities. ${ }^{5}$

With only 26 new trainees starting plastic surgery residencies each year in Canada, it can be challenging for small surgical programs like plastic surgery to accommodate parental leave. Starting a family during residency can be complicated and situationally dependent, and it is important to understand the impact of the environment on this life event by investigating the experiences of residents and recent graduates across Canada. This study aimed to report the experiences, attitudes and perceived support of Canadian plastic surgery residents and surgeons with respect to pregnancy and parenting during training.

\section{SURVEY}

Previously developed scales in the area of pregnancy and parental leave during residency in Canada and the United States were identified through 
a literature search. These studies were analyzed for strengths and weaknesses in terms of survey development, recruitment and data collection. A focus group then determined the variables, potential concepts and themes that should be extracted from the identified surveys. Selected questions were reviewed for redundancy and the number of items was reduced accordingly. Content validity and pretesting for format and composition evaluation were performed by external appraisal by surgical residents in other specialties and by all authors. On the basis of the results of this process, 2 surveys were developed: 1 targeted to staff surgeons and the other to residents and recent graduates. There were no "forced answers" (i.e., any question could be skipped).

Participants were invited via email to participate in the survey. The survey for residents and recent graduates was sent to all current plastic surgery residents and recent graduates (graduating year between 2014 and 2018). The staff survey was sent to all plastic surgeons registered with the Canadian Society of Plastic Surgeons, including plastic surgery program directors. Both surveys were sent to all plastic surgery programs in Canada. Each email message contained a brief description of the study and a link to a LimeSurvey web page, where there was a detailed outline of the study (the design, purpose and steps taken to maintain anonymity). This introductory page explained that participation was voluntary and that no compensation would be offered. Consent was implied when respondents submitted their completed survey. Respondents could exit the survey at any time.

Given the small size of plastic surgery training programs and the sensitive nature of this topic, data for recent graduates were amalgamated with data for current residents to protect the identity of respondents. Data were immediately anonymized and responses were combined; the responses of individual participants were not available to the study administrators.

The survey, the distribution method and the steps to ensure participant anonymity were designed in consultation with and approved by the Hamilton Integrated Research Ethics Board (HiREB 3446).

A total of 123 residents and recent graduates and 134 staff surgeons completed the survey. The demographic characteristics of respondents are presented in Table 1. Among female respondents, $21 \%$ of residents and $18 \%$ of staff surgeons gave birth to a child during residency. Of these respondents, $92 \%$ of residents and $86 \%$ of staff surgeons took maternity leave. Among male respondents, $24 \%$ of residents and $33 \%$ of staff surgeons fathered a child during residency. Of these respondents, $50 \%$ of residents and $0 \%$ of staff surgeons took parental leave (Fig. 1).

Seventy-two percent of residents and recent graduates did not want to become pregnant during residency, for a variety reasons. The most commonly reported

\begin{tabular}{|c|c|c|}
\hline \multirow[b]{2}{*}{ Characteristic } & \multicolumn{2}{|c|}{ No. $(\%)$ of respondents } \\
\hline & $\begin{array}{l}\text { Residents and } \\
\text { recent graduates } \\
n=123^{*}\end{array}$ & $\begin{array}{c}\substack{\text { Staff } \\
n=134^{*}}\end{array}$ \\
\hline \multicolumn{3}{|l|}{ Sex } \\
\hline Male & $58(50)$ & $44(35)$ \\
\hline Female & $58(50)$ & $81(65)$ \\
\hline \multicolumn{3}{|l|}{ Age, yr } \\
\hline $40-49$ & - & $43(32)$ \\
\hline $50-59$ & - & $33(25)$ \\
\hline \multicolumn{3}{|l|}{ Level of training } \\
\hline PGY1 or PGY2 & $23(20)$ & - \\
\hline PGY3 or above & $43(37)$ & - \\
\hline $\begin{array}{l}\text { Recently completed } \\
\text { residency }\end{array}$ & $51(44)$ & - \\
\hline \multicolumn{3}{|l|}{$\begin{array}{l}\text { No. of children during } \\
\text { residency }\end{array}$} \\
\hline \multicolumn{3}{|l|}{ Female respondents } \\
\hline 0 & 1 (8) & $33(83)$ \\
\hline 1 & $10(77)$ & $4(10)$ \\
\hline 2 & $1(8)$ & $2(5)$ \\
\hline$\geq 3$ & $1(8)$ & $1(3)$ \\
\hline \multicolumn{3}{|l|}{ Male respondents } \\
\hline 0 & $2(14)$ & $41(60)$ \\
\hline 1 & $7(50)$ & $11(16)$ \\
\hline 2 & $4(29)$ & 10 (15) \\
\hline$\geq 3$ & $1(7)$ & $6(9)$ \\
\hline \multicolumn{3}{|l|}{ No. of children } \\
\hline 0 & $79(68)$ & $13(10)$ \\
\hline 1 & $21(18)$ & 19 (15) \\
\hline 2 & $14(12)$ & $38(30)$ \\
\hline$\geq 3$ & $3(3)$ & $56(44)$ \\
\hline \multicolumn{3}{|c|}{$\begin{array}{l}\text { PGY = postgraduate year. } \\
\text { *Values do not add up to the total sample size for each category because some } \\
\text { questions were not answered by all respondents. }\end{array}$} \\
\hline
\end{tabular}

reasons were unwillingness to delay training and feeling unable to balance the demands of childcare and residency. Less frequently reported reasons were finances, lack of a suitable partner, fertility or no desire for children (at the time).

Of the residents and recent graduates who did become pregnant, $42 \%$ experienced complications. These included low amniotic fluid, placental insufficiency, first trimester bleeding and premature uterine contractions. No abortions were reported. Seven percent reported 1 or more miscarriages during residency training.

Of the program directors, $57 \%$ reported a formal parental leave policy specific to their plastic surgery program or the department of surgery. Of the residents and recent graduates, $38 \%$ reported that their program or surgical department had no formal parental leave policy and $48 \%$ were unsure whether a policy existed.

The responses of residents and recent graduates and of staff surgeons to questions regarding their opinions about 


\section{A}

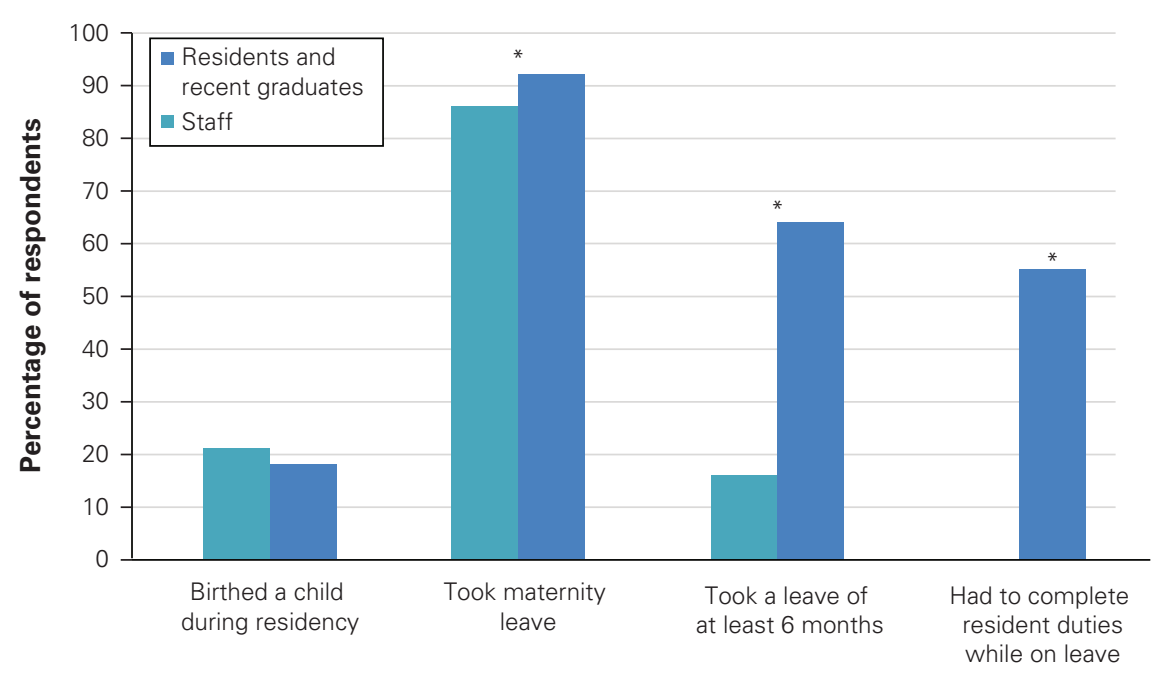

B

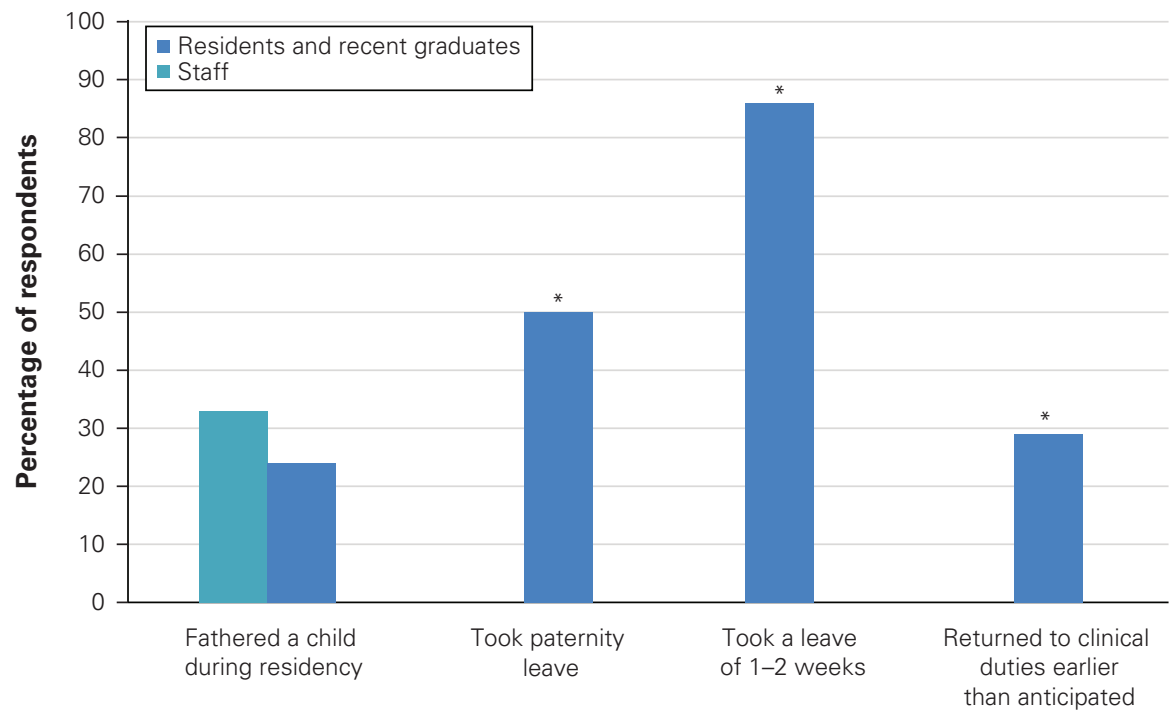

Fig. 1. Parental leave details reported by (A) female and (B) male residents and recent graduates and staff surgeons. *Data from respondents who birthed or fathered a child during residency.

pregnancy and parental leave during training are presented in Figure 2. Figure 3 outlines the beliefs of respondents regarding how parental leave affects the workload of others. The opinions of current residents and recent graduates on what should be included in a parental leave policy are reported in Figure 4. The component that respondents most frequently cited as being important to include in such a policy was the program's expectations concerning how far into pregnancy a resident would be expected to take call.

\section{Discussion}

The results of this study highlight the need for increased support for residents who wish to parent children during training. Additionally, the perceived confusion around program-specific policies emphasizes the need for open conversations and standardization of parental leave. Our findings suggest that although there may be improvements in the support provided by program directors to 
A

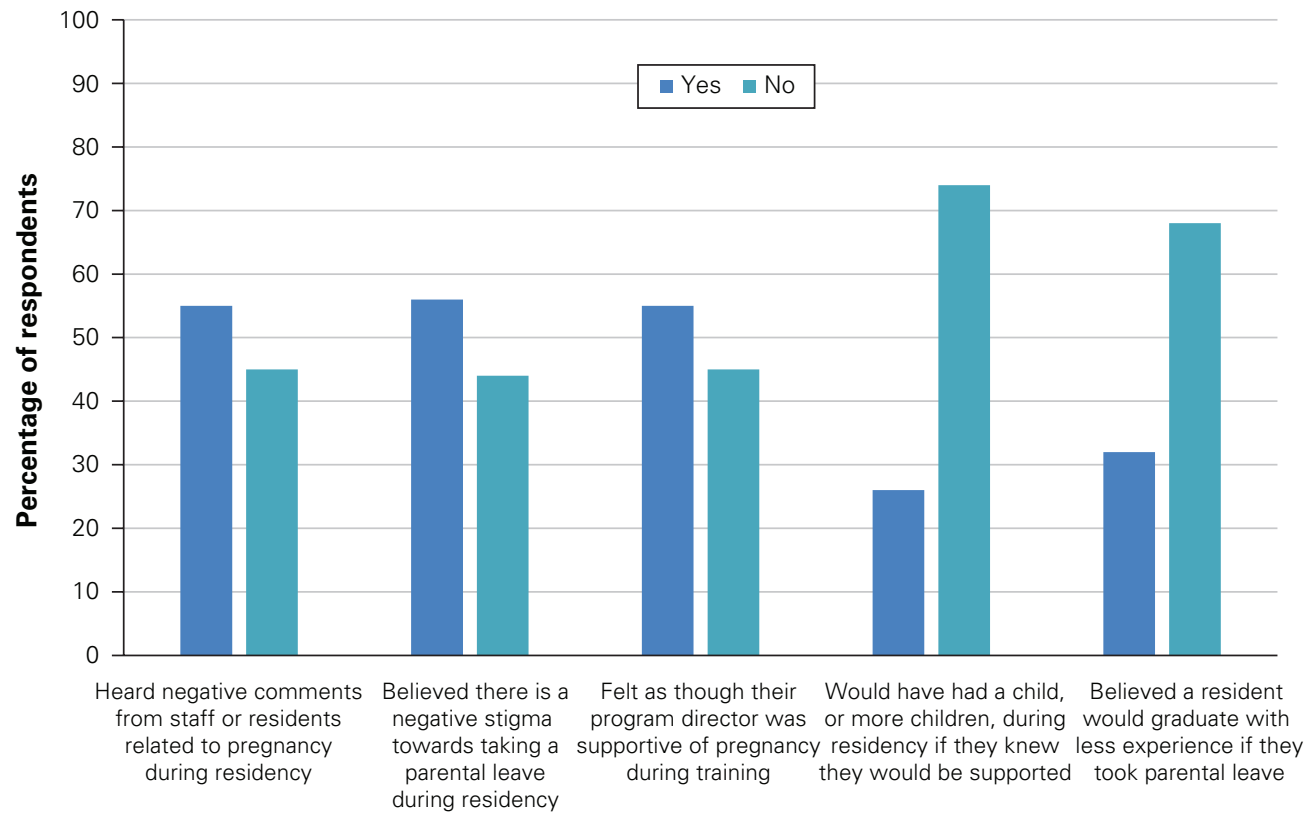

B

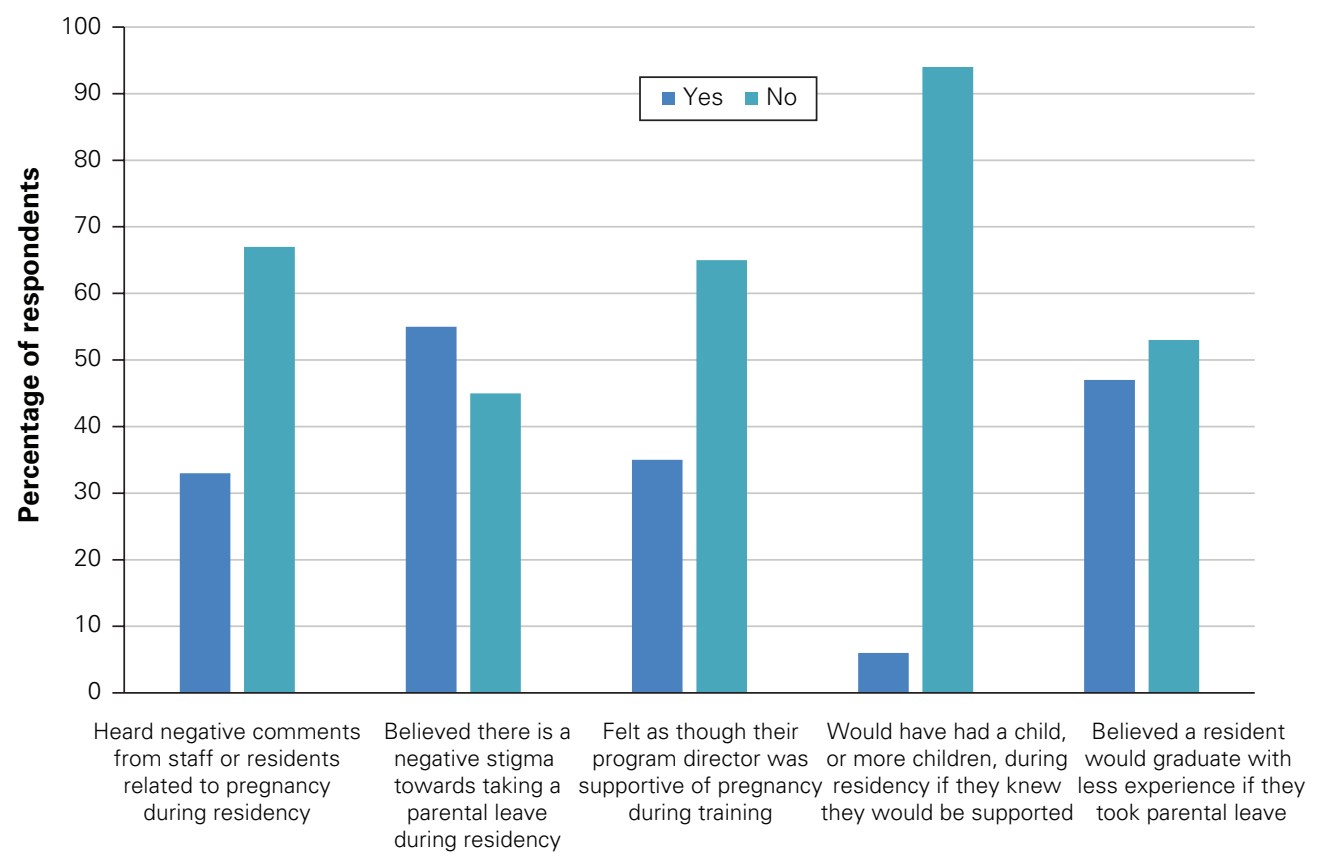

Fig. 2. Experiences of, and opinions on, parenting a child during residency among $(\mathrm{A})$ residents and recent graduates and (B) staff.

trainees who have children, negative attitudes toward pregnancy during residency persist in surgical culture.

Although a large proportion of respondents were unaware of policies related to pregnancy and parental leave, these policies exist in the professional association of residents' provincial contract. However, these policies are applied to all residents irrespective of their specialty. As such, these policies do not consider differences among 
A

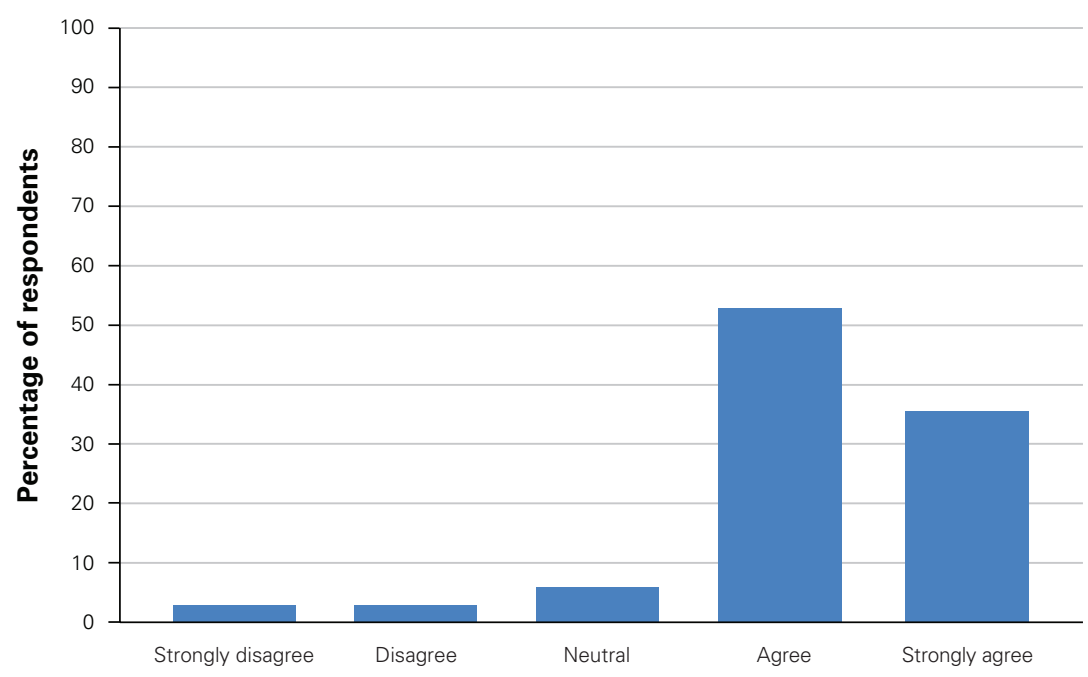

Response to statement: "My own workload would be negatively affected if another resident took a parental leave."

B

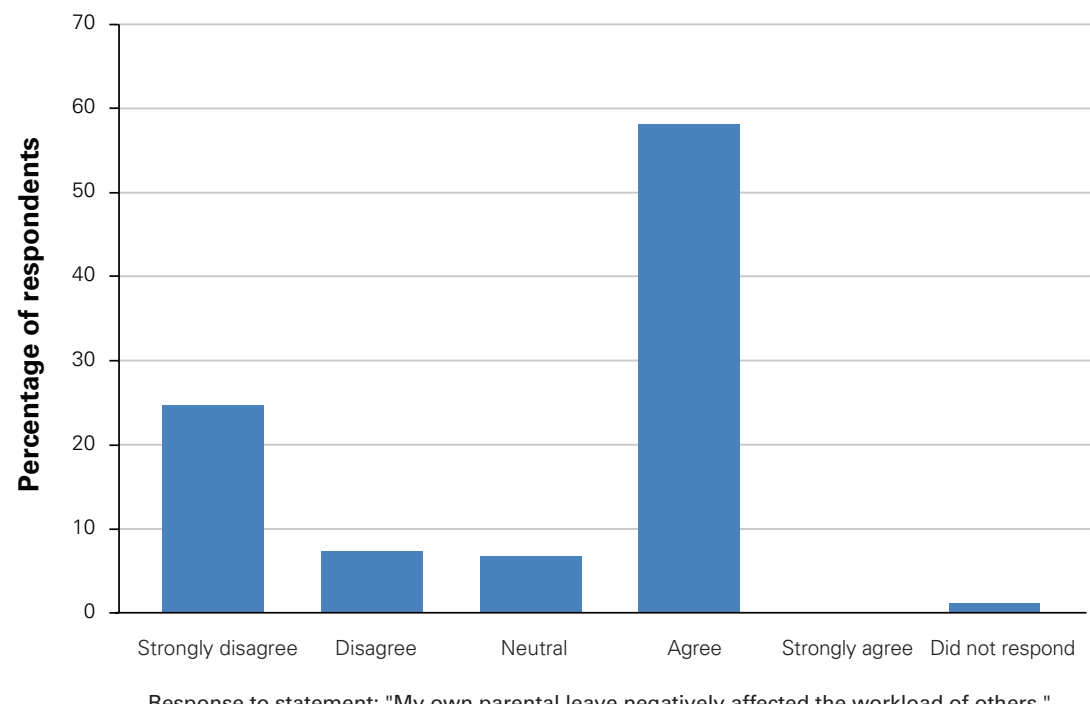

Response to statement: "My own parental leave negatively affected the workload of others."

Fig. 3. (A) Views of residents who were not pregnant during residency, on whether their own workload would increase if another resident took a parental leave. (B) Views of residents who were pregnant during residency, on whether their own pregnancy increased the workload of their coresidents.

programs. The typical plastic surgery residency program is small, with an average of 10 residents per training program; therefore, the absence of 1 resident can have a substantially greater impact in a plastic surgery program than in a larger program of another specialty. The development and implementation of pregnancy and parental leave policies for plastic surgery residency programs would not only help those residents remaining at work, but would also ensure the safety of pregnant residents and their unborn children.

Limitations include the potential for responder bias, the untested reliability of the survey, and the fact that the survey was available only in English. Additionally, we acknowledge that this is a multifaceted topic with several 


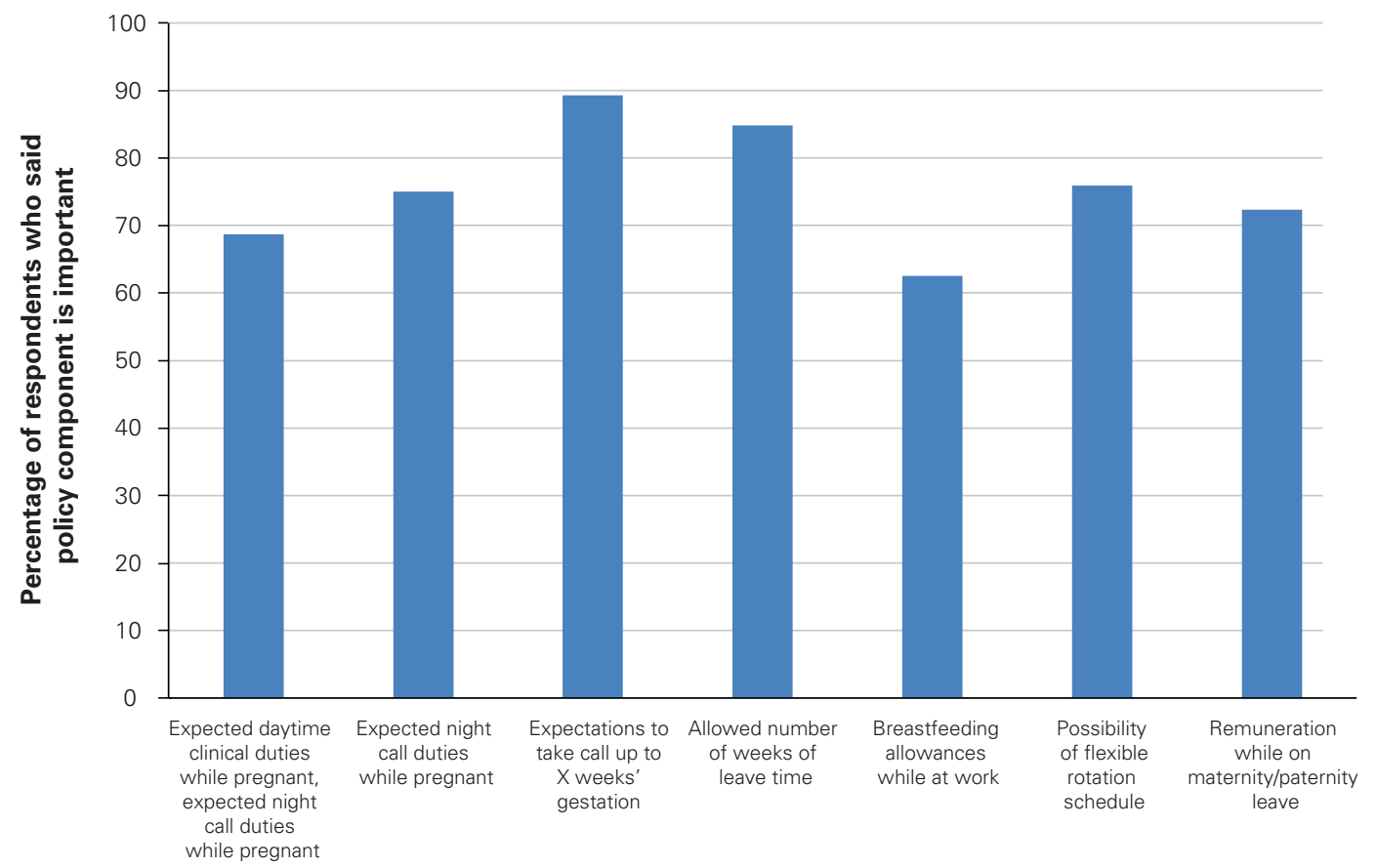

Policy component

Fig. 4. Components of parental and maternity leave policies felt to be important by residents and recent graduates.

relevant issues and challenges that were not all addressed in this study. Nevertheless, we hope that this study will initiate more conversation and action on this topic.

Acknowledgements: The authors thank Drs. Annie Wang, Mona Al-Taha and Mike Stein, and members of the Canadian Plastic Surgery Research Collaborative, for helping to distribute the survey and collect the results.

Affiliations: From the Department of Surgery, Division of Plastic Surgery, McMaster University, Hamilton, Ont. (Augustine, Rizvi, Dunn, Murphy, Avram, Voineskos); the Department of Surgery, Division of Plastic Surgery, University of Montreal, Montreal, Que. (Retrouvey, Efanov); the Department of Surgery, Division of Plastic Surgery, McGill University, Montreal, Que. (Alhalabi); the Division of Plastic Surgery, University of Toronto, Toronto, Ont. (Retrouvey); the Department of Surgery, University of Calgary, Calgary, Alta. (Steve).

Competing interests: None declared.

Contributors: All authors contributed substantially to the conception, writing and revision of this article and approved the final version for publication.

\section{References}

1. Canadian Post-M.D. Education Registry. 2018-2019 annual census of post-M.D. trainees. Ottawa: Canadian Post-M.D. Education Registry; 2019. Available: https://caper.ca/sites/default/files/pdf/annual -census/2018-19-CAPER_Census_en.pdf (accessed 10 Sept. 2019).

2. Moyser M, Milan A. Insights on Canadian society: fertitility rates and labour force participation among women in Quebec and Ontario. Ottawa: Statistics Canada; 2018. Available: https://www150.statcan.gc.ca/n1/ pub/75-006-x/2018001/article/54976-eng.htm (accessed 10 Sept. 2019).

3. Merchant S. Pregnancy among residents enrolled in general surgery (PREGS): a survey of residents in a single Canadian training program. Can 7 Surg 2011;54:375-80.

4. Furnas HJ, Li A, Garza R, et al. An analysis of differences in the number of children for female and male plastic surgeons. Plast Reconstr Surg 2019;143:315-26.

5. Task Force on the Accommodation of the Pregnant Physician. Medicine and motherhood: Can we talk?: A consensus statement. Vancouver: Physician Health Program of British Columbia; 2010. Available: https://www.physicianhealth.com/sites/default/files/images/Medicine AndMotherhood2010.pdf (accessed 7 Oct. 2019). 